\title{
Collision Avoidance Law Using Information Amount
}

\author{
Seiya Ueno and Takehiro Higuchi \\ Yokohama National University \\ Japan
}

\section{Introduction}

The collision avoidance control has been one of the key technology for future transportation. Recently, many unmanned systems are developed in shapes of robots, cars, ships, aircraft, etc. In these environments, proper navigation and control systems including collision avoidance is needed. This paper is on collision avoidance control law for air vehicles under uncertain information. The control law uses information amount as one of the physical parameter for control system.

In the field of guidance, navigation, and control, collision avoidance of automated transportation system has been one of main interest of researchers. Many researches started from collision avoidance of ships (Ciletti et al., 1997) where collision avoidance has been one of the problems due to the increasing demand for the naval transportations. Wide varieties of studies on collision avoidance are treated in fields of robots (Fukuda \& Kubota, 1999), cars (Hiaoka et al., 2009a, 2009b) and satellites. Some of these researches treat avoidance problems with the formation control which requires the cooperative information control (Slater et al., 2006; Stipanovic et al., 2007).

In the field of aeronautics, the Traffic alert and Collision Avoidance System (TCAS) has been one of the references for the collision avoidance. TCAS exchanges the information of aircrafts and advises the aircraft to avoid in vertical direction. For the conflicts in collision avoidance control, Frazzoli et al. (2001) have shown feasible strategy to treat the conflict problem. Gates (2009) has proposed rule-based collision avoidance control strategies for real-time online collision avoidance. Miele et al. (2010) has proposed collision avoidance control for case of abort landing with low computational load which can be calculated by on-board computer.

Conventional avoidance problems assume that all information about avoidance (intruders and environments) is certain. Therefore, control law is designed based on certain information. However in real cases, all information may not be correct and most of it is uncertain. These uncertainty of information differes by the relative position of the evader and intruder or the absolute position of intruder. There has been no research on control law to deal with uncertain information. This paper proposes control law that treats uncertain information. New parameters quantifying information amount are defined for this purpose. The proposed control law provides new performance by enabling the aircraft to obtain information and to check the certainty of the information.

Two different cases of numerical simulations are used to investigate the usage of the information amount. The first case defines the problem as the uncertainty of the information changes by the relative position of the evader and the target. The problem treats the case 
where the amount of infomation changes by relative position, for example, the flight in fog or smoke. The information is clearer as the evader gets closer to the fog. These uncertainties are quantified and used as parameters for collision avoidance control law. The second case defines the problem as the uncertainty of the infomation is given as absolute position. The infomation can or cannot be obtained by the position itself, for example, the flight around urban buildings or moutains. In both cases, the information amount is obtained from focused area assigned by the user. Using the information amount, the control law is designed for safer flight of the air vehicles.

\section{Information amount}

In this study, amounts of information are treated as parameters for the control law. First, the focused area : $S_{E}$ is treated as the region of the area that the user focuses. This area can be large if the vehicle is moving fast or very small if the vehicle is in urban area moving very slowly. The cleared area : $\mathrm{S}_{\mathrm{C}}$ is the area where the information are certain. In the cleared area, all of the infomation is available, meaning if there is an intruder in that region, the evader can obtain all the infomation of the intruder. In the other hand, the blurred area : $S_{B}$ is the area where infomation is uncertain.

From these parameters of the areas, the infomation amount is derived qunatatively as physical value to be used. One of the important factor used in this paper is information localization $: I_{L}$ which is calculated from the amount of cleared area out of the focused area. The $I_{L}$ is,

$$
I_{L} \equiv S_{C} / S_{E}
$$

and the schematic image of this areas are shown in Fig.1.

Another important factor for the information amount is information acquisition requirement $: I_{R}$ which is requirement of the $I_{L}$ for safe flight. The evader selects the proper amount for the vechicle to obtain. The higher the value is, more infomation have to be obtained. In the other hand, evader have to move more when the value is high. This amount can be changed by the requirement of the user.

The infomation amounts can be changed according to the users request and experience. If the evader is moving fast, the $S_{E}$ might be large but the $I_{R}$ could be small. If the evader is trying to turn the corner, $S_{E}$ might be small but the $I_{R}$ could be very large. These amounts are similar to that of human sence of avoiding the dangers, which makes this amount unique and useful for colision avoidance.

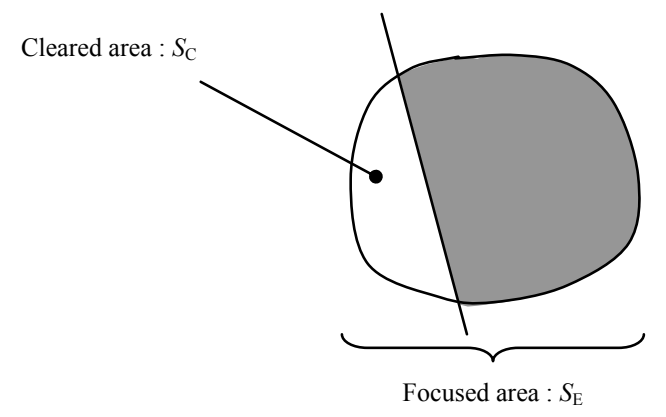

Fig. 1. Schematic image of information amounts. 


\section{Collision avoidance law}

The total system of collision avoidance law in this paper consists of three types of control laws. They are actual collision avoidance, information gathering, and cource keeping. They are switched by the risk of collision and amount of infomation obtained. All of the simulation are in 2-dimensions and either acceralation or angular velocity of the vehicle is used as input variables. Fig. 2 shows the basic definition of variables and constants used in this paper.

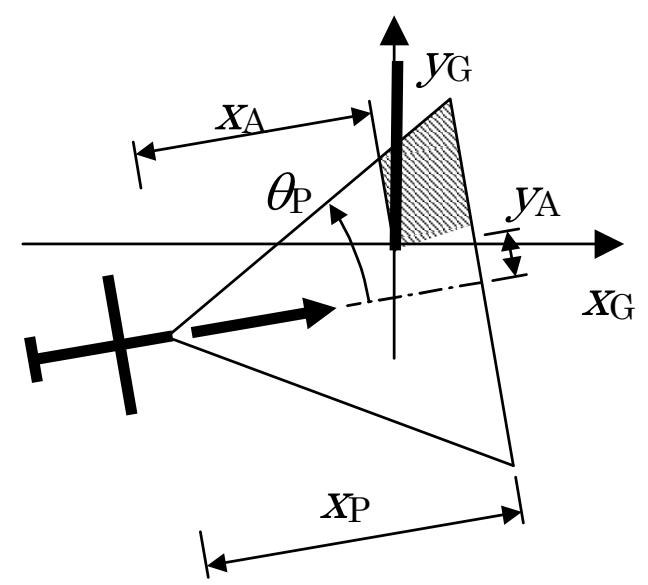

Fig. 2. Definitions of variables and constants.

The risk of collision is described numerically for collision avoidance control law. Two values are introduced in this paper. One is Range to Closest Point to Approach: $R_{C P A}$, which is the minimum range between two vehicles when their velocities and directional angles are kept at present value. The closest point is shown in Fig.3. This value indicates the future risk of collision. The other is Time to Closest Point to Approach: $T_{C P A}$, which is time to the range between two vehicles is $R_{C P A}$. Even if $R_{C P A}$ is small, it is not necessary to avoid quickly

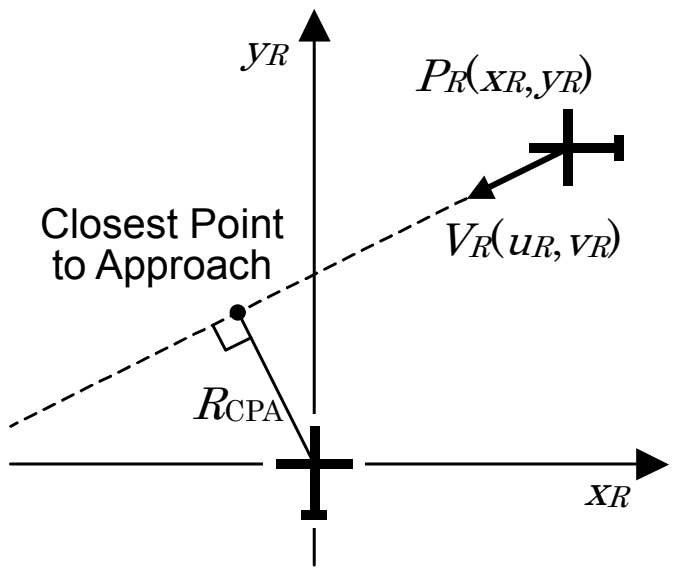

Fig. 3. Image of closest point to approach. 
when $T_{C P A}$ is large, in the othere hand, when the $T_{C P A}$ is small, the evader have to start the motion very quickly. The collision avoidance control law is derived from the combination of these two risk functions.

$R_{C P A}$ and $T_{C P A}$ are driven using the relative position and velocity in the body fixed coordinate shown in Fig.3. The $T_{C P A}$ is derived as,

$$
T_{C P A}=-\left(x_{R} u_{R}+y_{R} v_{R}\right) /\left(u_{R}^{2}+v_{R}^{2}\right)
$$

Then, the relative position and range at closest point of approach is given as,

$$
\begin{gathered}
\left(\begin{array}{l}
x_{C} \\
y_{C}
\end{array}\right)=\left(\begin{array}{l}
x_{R} \\
y_{R}
\end{array}\right)+T_{C P A} \cdot\left(\begin{array}{l}
u_{R} \\
v_{R}
\end{array}\right) \\
R_{C P A}=\sqrt{x_{C}^{2}+y_{C}^{2}}
\end{gathered}
$$

The risk function is defined as the following equation.

$$
\varphi \equiv R_{0}^{2} /\left(R_{0}^{2}+R_{C P A}^{2}\right)
$$

where $R_{0}$ represents the safety range. Collision will occur when $\varphi=1$. When the minimum range between two vehicle is equal to $R_{0}, \varphi=0.5$. The collision avoidance control law is designed to reduce $\varphi$ less than 0.5 in a period of $T_{\mathrm{CPA}}$. These parameters that show the risk of collisions are used for collision avoidance. When the risk is high, the direct collision avoidance control is activated to avoid the collision. One of the examples of collision avoidance control law is described in the following.

The collision avoidance control law satisfies the following equation.

$$
\dot{\varphi}=-\varphi / T_{C}
$$

$T_{C}$ is time constant that is derived from the following requirement.

$$
T_{C}=-T_{C P A} / \ln \left(2 \varphi_{0}\right)
$$

where $\varphi_{0}$ is the initial value of risk function. The left hand side of Eq. (6) can be derived from the derivative of Eq. (5) and eliminating the effect of the angular velocity of the evader. The effect of the angular velocity is momentary, where they return to their original values after the avoidance. Let the absolute velocity and angle of direction of the evader be, $V_{0}$ and $y_{0}$, and the intruder be, $V_{1}$ and $y_{1}$, the relative velocity of the vehicles can be expressed as,

$$
\left(\begin{array}{c}
u_{R} \\
v_{R}
\end{array}\right)=\left(\begin{array}{c}
y_{R} \\
-x_{R}
\end{array}\right) \dot{\psi}_{0}+\left(\begin{array}{cc}
\cos \left(\psi_{1}-\psi_{0}\right) & 1 \\
\sin \left(\psi_{1}-\psi_{0}\right) & 0
\end{array}\right)\left(\begin{array}{c}
V_{1} \\
V_{0}
\end{array}\right)
$$

The first factor of the right hand side is the angular velocity of the evader, so by eliminating this factor, the following can be derived by taking the derivative of the relative velocity.

$$
\frac{d}{d t}\left(\begin{array}{c}
u_{R} \\
v_{R}
\end{array}\right)=\left(\begin{array}{c}
v_{R} \\
-u_{R}
\end{array}\right) \dot{\psi}_{0}
$$


As total, the angular velocity for collision avoidance can be derived as,

$$
\left(\omega_{0}\right)_{A}=\frac{1}{p_{C} v_{R}-q_{C} u_{R}} \cdot \frac{R_{0}^{2}}{2 \varphi T_{A}}
$$

where,

$$
\begin{aligned}
& p_{C}=x_{C} p_{x}+y_{C} p_{y} \\
& q_{C}=x_{C} q_{x}+y_{C} q_{y} \\
& p_{x}=v_{R}\left(-u_{R} p_{o}-v_{R} q_{i}\right) /\left(u_{R}^{2}+v_{R}^{2}\right) \\
& q_{x}=u_{R}\left(2 v_{R} p_{i}-y_{R}\left(u_{R}^{2}+v_{R}^{2}\right)\right) /\left(u_{R}^{2}+v_{R}^{2}\right) \\
& p_{y}=v_{R}\left(2 u_{R} p_{i}-x_{R}\left(u_{R}^{2}+v_{R}^{2}\right)\right) /\left(u_{R}^{2}+v_{R}^{2}\right) \\
& q_{y}=u_{R}\left(v_{R} p_{o}-u_{R} p_{i}\right) /\left(u_{R}^{2}+v_{R}^{2}\right) \\
& p_{i}=x_{R} u_{R}+y_{R} v_{R} \\
& p_{o}=x_{R} v_{R}-y_{R} u_{R}
\end{aligned}
$$

This collision avoidance control law activates when the risk is high. In other words, other 2 control laws, infomation gathering and course keeping laws are used when the risk is low. In the following 2 sections, the different types of infomation gathering control laws are introduced depending on the difference of the uncertainties up ahead. The course keeping control law is used to keep the original course, which is not important in this paper, so will not be explained in details.

\section{Uncertainty depending on relative position}

The information gathering control with uncertainty depending on relative position is introduced in this section. The uncertainty depending on relative position stands for the cases where the infomation that can be obtained are defined as function of relative distance to an uncertainty. This is applicable for the flights in the fog or smokes where the uncertainty differs by the distance, closer you are, clear infomation you can obtain. The control target is fixed wing aircraft and the control input is angular velocity. The control law is designed from fuzzy logic to realize the fuzziness of the infomation. First, the additional parameters of the uncertain infomation is explained in this section. Followed by the control law and control results.

\subsection{Uncertain parameters}

The control law uses additional parameters for infomation in this section. The basic parameters were $I_{L}$ and $I_{R}$ which was explained in section 2. The following parameters quantifying uncertainty are added to design the control law dealing with uncertain information. Uncertainty of information depends on the target existence and location. Therefore, first, uncertainty parameters are defined separately. Then, the uncertainty coefficient for the control law is obtained from the uncertainty parameters for existence and location. 


\subsubsection{Information probability $-I_{P}$}

Infomation probability is a parameter describing the probability, possibility or likelihood of the target existence. $I_{P}$ takes a fixed value from 0 to 1 and is assigned by the user before avoidance. When $I_{P}=0$, there is no probability of existence. On the other hand, the target existence is certain when $I_{\mathrm{P}}=1$.

\subsubsection{Information clarity $-I_{C}$}

Infomation clarity is a parameter describing the clarity of target existence. $I_{C}$ varies with the quantity of information. When $I_{C}=0$, there is no information about existence. On the other hand, information on target existence becomes clear when $I_{C}=1$. When the information has uncertainly, $I_{C}$ varies from 0 to 1 with relative distance between the target and evader. For example, $I_{C}$ varies as shown in Fig. 4 when visibility is obscured by fog. Information becomes clear $\left(I_{C}=1\right)$ at a certain distance and worsens gradually with further distance. No information is provided $\left(I_{C}=0\right)$ beyond a certain distance. Figure 1 shows an environment when visibility is just barely secured at $5000 \mathrm{~m}$. The target is found definitely when the relative distance is less than $4000 \mathrm{~m}$. As the relative distance increases, visibility worsens gradually and the target cannot be found when the relative distance exceeds $5000 \mathrm{~m}$.

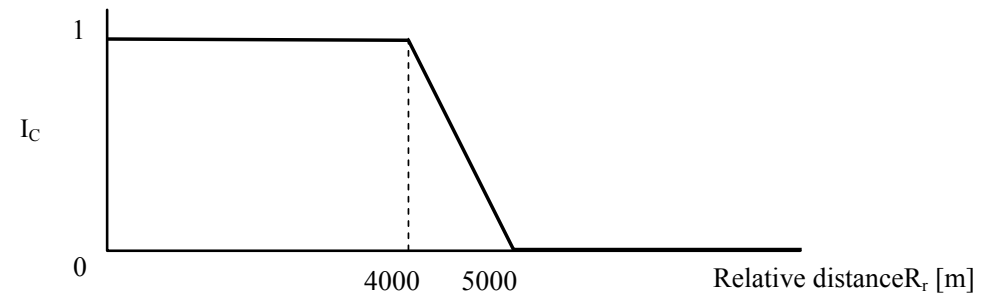

Fig. 4. Information clarity : The figure shows the case when the visibility is $4000-5000[\mathrm{~m}]$. The information is clear when the distance is lower than $4000[\mathrm{~m}]$ and cannot be obtained when the distance is over $5000[\mathrm{~m}]$. The information clarity changes linearly between two regions.

\subsubsection{Information truth $-I_{T}$}

Infomation truth is a parameter describing truth of the target existence. The value determines whether the target exists or not. It takes a value of either 0 or 1 . When $I_{\mathrm{T}}=0$, there is no target. On the other hand, when $I_{\mathrm{T}}=1$, there is a target.

\subsubsection{Information location accuracy $-I_{A}$}

Infomation location accuracy is a measure of the area in which the target exists. For example, in a 2D model, $I_{\mathrm{A}}$ is a circle with a radius of arbitrary length. As shown in Fig. 5, it is assumed that $I_{\mathrm{A}}$ depends on the radius of the zone containing the target. $I_{\mathrm{A}}$ takes a value between 0 (existence zone is vast) to 1 (existence zone is very small). In summary, the existence zone is the domain where the target may exist.

\subsection{Application to control law}

The control law using the uncertain infomation is introduced. From the viewpoint of complexity and difficulty, it is wrong to design a whole new control law adopting 


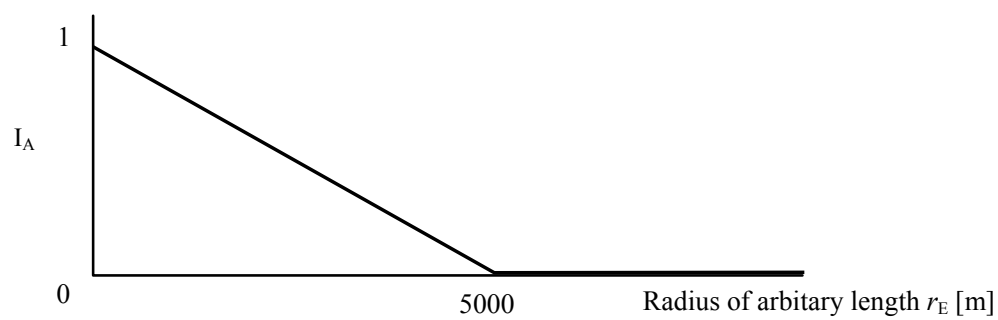

Fig. 5. Information location accuracy: The figure shows that the unknown target is within $5000[\mathrm{~m}]$ of radius with highest possibility at the center.

uncertainty. Therefore, the control law to deal with uncertainty simply by introducing technique to the conventional control law with only minor modification is proposed.

\subsubsection{Uncertainty coefficient}

As a first step in designing a control law to deal with uncertainty, the uncertainty coefficient $k$ based on the uncertainty parameters of existence and location $\left(I_{P}, I_{C}\right.$ and $\left.I_{T}\right)$ is brought in. $k$ is a function of these parameters expressed as,

$$
k=f\left(I_{P}, I_{C}, I_{T}\right)
$$

As shown in Fig. 6, $k$ is a coefficient introduced for the following reasons. Under the conventional control law, target information is certain and the evader flies a course for either target existence or target absence. On the other hand, when the information is uncertain, the evader flies somewhere between target existence and target absence. It is assumed that $k$ has three components as follows, depending on relative distance: part based on original estimate (corresponding to $I_{C}=0$ ), part based on information that gradually becomes clear (corresponding to $0<I_{C}<1$ ), and part based on clear information (corresponding to $I_{\mathrm{C}}=1$ ).

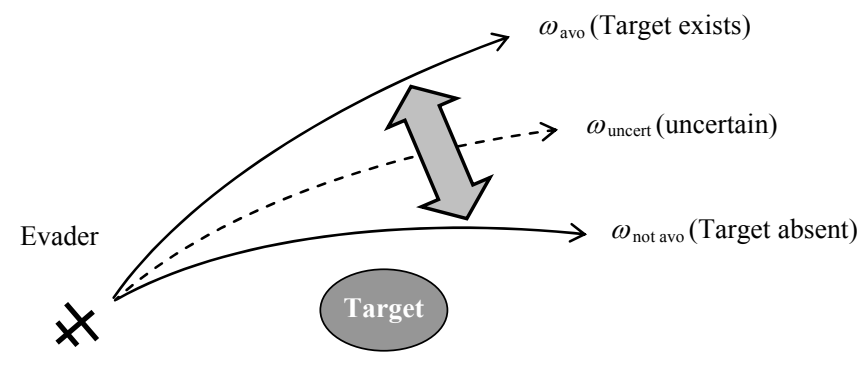

Fig. 6. The trajectory of uncertain condition and input.

In the situation in Fig. 4, when each uncertain parameter is given, the value of $k$ becomes as shown in Fig. 7. $k$ takes a constant value depending on $I_{P}$ when $R_{\mathrm{r}}>5000$, because the amount of information quantity does not vary in this condition. Information gradually 
becomes clear when $4000<R_{\mathrm{r}}<5000$. Variation of $I_{\mathrm{C}}$ reflects variation of $k . k$ takes either 0 or 1 depending on only $I_{\mathrm{T}}$ when $R_{\mathrm{r}}<4000$, because the information becomes certain. $I_{\mathrm{T}}$ is finally decided whether the target exists or not. However, if it is assumed that $I_{\mathrm{T}}$ approaches a true value gradually as the information becomes clear, $k$ is determined in real time. In such a circumstance, control input (angular velocity) $\omega_{\text {uncert }}$ takes a value between target existence and target absence. Therefore $\omega_{\text {uncert }}$ is expressed by the following equation using $k$,

$$
\omega_{\text {uncert }}=k \omega_{\text {avo }}+(1-k) \omega_{\text {notavo }}
$$

where $\omega_{\text {avo }}$ and $\omega_{\text {notavo }}$ are the control input to the evader for target existence and target absence, respectively. Avoidance control depends on the area where the target may exist, thus $\omega_{\text {avo }}$ is a function of $I_{A}$.

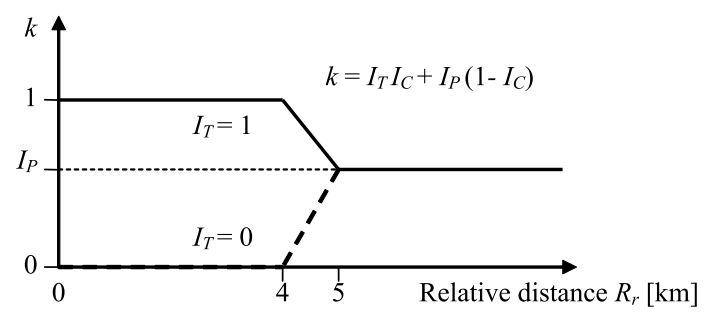

Fig. 7. Image of uncertainty coefficient with uncertain information.

\subsubsection{Information acquisition requirement}

The $I_{R}$ explained in section 2 is now brought in for the control. As explained in section 2, the $I_{R}$ is the requirement of $I_{\mathrm{L}}$ by the user. $I_{\mathrm{R}}$ represents the degree of need to obtain information on the focused area. $I_{R}$ is determined by the user and takes a value from 0 to 1 . When $I_{R}=0$, there is no requirement. On the other hand, all information is required when $I_{R}=1$. As shown in Fig. 8 , the clear region in the focused area becomes small as $I_{R}$ approaches 0 . In contrast, as $I_{R}$ approaches 1 , the cleared part becomes large. If the conventional control law is modified by introduction of the uncertainty coefficient, $k$, and the information acquisition requirement, $I_{R}$, design of a new control law to deal with uncertainty is comparatively easy.
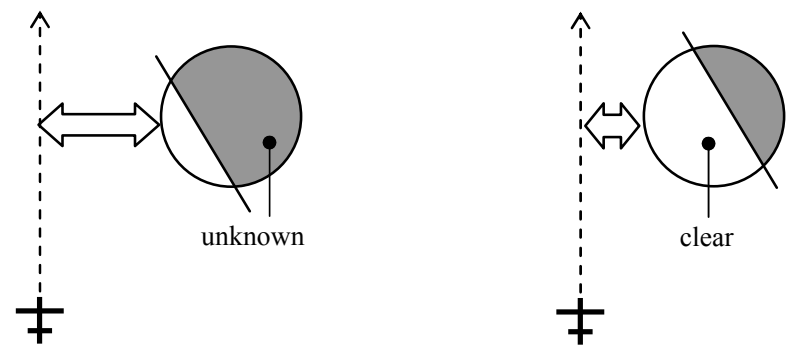

Fig. 8. Image of information acquisition requirement. Left figure shows the case where $I_{R}$ is small (vehicle passes in far region from the focused area), right figure shows the case where $I_{R}$ is large (vehicle passes in closer region from focused area). 


\subsection{Simulation result of relative position - in-fog problem}

An example of avoidance problem is uncertainty of information defined in the relative coordinate (body fixed) system is shown. The problem is assumed to be in-fog problem, where there is area where the information is uncertain upahead.

\subsubsection{Statement of problem}

The evader cannot see the target beyond a certain distance because visibility is obscured by an obstacle like fog. The problem is defined as two-dimensional in the horizontal plane. The evader flies on a straight course with constant velocity towards a target that may exist in existence zone as shown in Fig. 9. Visibility is defined as a function of relative distance from the evader. When the relative distance is smaller than a certain distance, for example $4000 \mathrm{~m}$, the evader can see the target clearly. However, the evader cannot see the target when the relative distance is larger than a certain distance, for example $5000 \mathrm{~m}$. Visibility changes gradually between these two areas. The information clearness $I_{C}$ is defined depending on the relative distance to the target as for visibility as explained in Fig.4. The target is close to evader's course, but information about existence and position are uncertain. Therefore, the target existence is given as the information probability, $I_{P}$, and the position is given as the target existence zone (circlular region with radius of $r_{E}$ ).

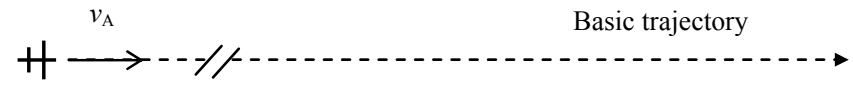

Evader
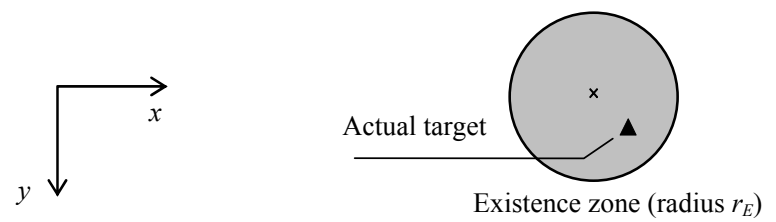

Fig. 9. Image of the problem, the target is assumed to be inside the fog where, the information obtained from evader is uncertain.

\subsubsection{Initial conditions and requirement}

The initial position of the evader and target existence zone are shown in Fig. 9. Other constants are shown in Table 1. Figure 4 is used for $I_{C}$. Also the required separation between the evader and the target is set from $3000 \mathrm{~m}$ to $4000 \mathrm{~m}$.

\subsubsection{Initial conditions and requirement}

Figures 10 and 11 show the avoidance trajectory and angular velocity, respectively. Solid lines represent the results for the proposed control law; dashed lines represent the results for the conventional control law. The figure shows two cases for the conventional control law: avoidance with correct information; and avoidance with incorrect information where target appears suddenly without information.

The avoidance trajectories in Fig. 10 show that avoidance using the conventional control law with incorrect information causes significant delay because the evader does not avoid until the target is found. On the other hand, the avoidance trajectories produced by the proposed 


\begin{tabular}{|c|c|c|c|c|}
\hline Parameters & Symbols & Values & $\begin{array}{c}\text { Uncertain } \\
\text { parameters }\end{array}$ & Values \\
\hline Velocity & $v_{A}$ & $250[\mathrm{~m} / \mathrm{s}]$ & $I_{P}$ & 0.25 \\
\hline Initial position & $r_{E}$ & $1000[\mathrm{~m}]$ & $I_{C}$ & Shown in Fig. 4 \\
\hline $\begin{array}{c}\text { Radius of existence } \\
\text { zone }\end{array}$ & & $(25000,2000)$ & $I_{T}$ & 1 \\
\hline $\begin{array}{c}\text { Center of existence } \\
\text { zone }\end{array}$ & $(25500,2500)$ & $I_{R}$ & Shown in Fig.5 \\
\hline $\begin{array}{c}\text { True position of } \\
\text { target }\end{array}$ & & & $0.2,0.9$ \\
\hline
\end{tabular}

Table 1. Parameters and constants for the simulation.

control law depend on the value of $I_{R}$, because $I_{R}$ indicates the degree of necessary information. When $I_{R}$ is large, the evader must fly closer to the target and does not take early avoidance. Therefore, two stage avoidance occurs when $I_{R}=0.9$. The first stage is infomation gathering based on current infomation and information acquisition; the second stage is avoidance after finding the target. Figure 11 shows the angular velocities for avoidance. Information uncertainty reduces the sudden and severe avoidance that occurs using the conventional control law with incorrect information.

Both of the figures show that the proposed control law was able to increase the safetiness and reliability of the flight in uncertain information defined in relative position from the evader.

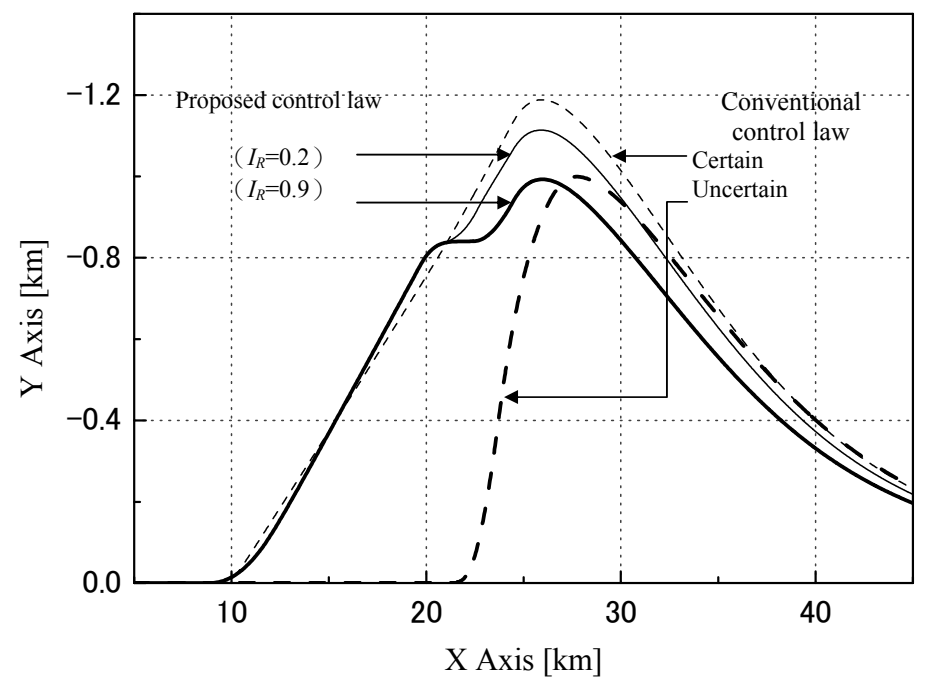

Fig. 10. Avoidance trajectory of the cases with/without proposed control law using uncertain parameters $\left(I_{P}=0.25\right)$. 


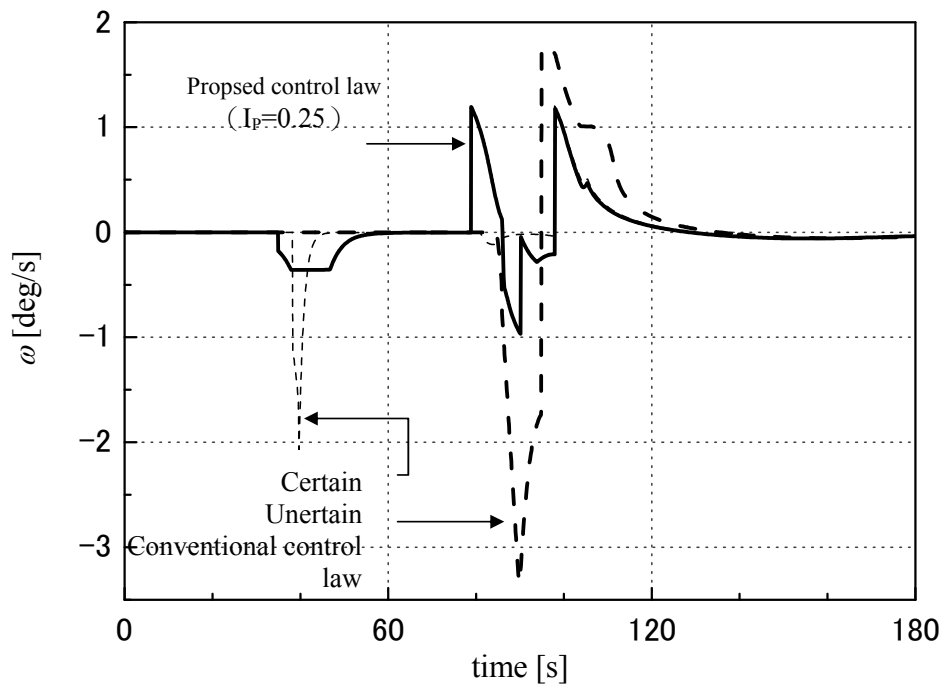

Fig. 11. Angular velocity for avoidance of the cases with/without proposed control law using uncertain parameters $\left(I_{P}=0.25\right)$.

\section{Uncertainty depending on absolute position}

In this section, the uncertainty depending on absolute position is treated. Different from the uncertainty that differs by relative position as explained in section 4 , the information does not change due to the environment. For example, when aircraft is going around a mountain or a helicopter going around the buildings, the information does not change due to relative position.

The information parameters $I_{L}$ and $I_{R}$ which was explained in section 2 is used in this section to see the effect of the information amount. The collision avoidance control explained in section 3 is brought in when the vehicle enters critical condition, otherwise the following either infomation gathering control or course keeping control takes place.

\subsection{Design of information gathering control law}

Design of information gathering control law is derived using a model in Fig.12 and Fig.13. Figure 12 is the vehicle in the ground fixed coordinate and Fig.13 shows the vehicle in body fixed coodinate. Focused area depends on the speed and direction of the vehicle, so the distance $x_{P}$ can be set by the users descision. The angle $\theta_{P}$ also depends on the level of safety which can be chosen. The area of focused area is given as follows.

$$
S_{E}=x_{P}^{2} \cdot \tan \theta_{P}
$$

The shadow area cannot be seen from the vehicle. So, the area of shadow area is assumed as the following equation using the focused area.

$$
S_{B}=\frac{1}{2}\left(x_{P}^{2}-x_{A}^{2}\right) \cdot\left(\tan \theta_{P}-\tan \theta_{A}\right)
$$




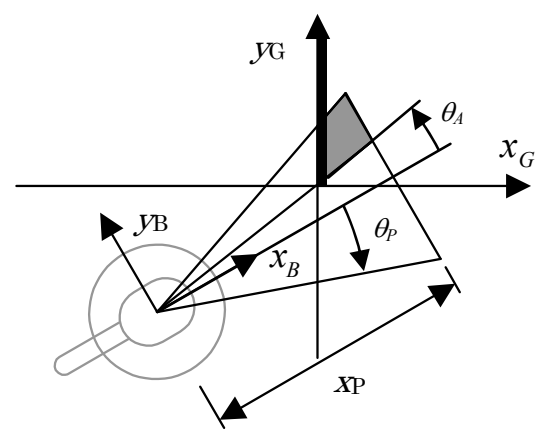

Fig. 12. Vehicle in ground fixed coordinate system.

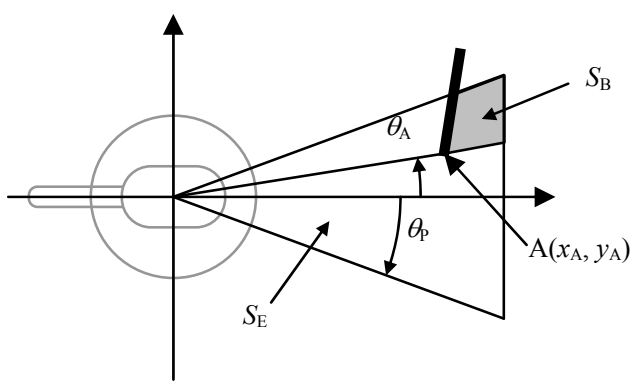

Fig. 13. Vehicle in body fixed coordinate system.

where,

$$
\tan \theta_{A}=y_{A} / x_{A}
$$

$x_{A}$ and $y_{A}$ are relative coordinate in the body fixed frame. The time derivatives of $x_{A}$ and $y_{A}$ are given as follows.

$$
\frac{d}{d t}\left(\begin{array}{l}
x_{A} \\
y_{A}
\end{array}\right)=\left(\begin{array}{c}
y_{A} \\
-x_{A}
\end{array}\right) \omega_{0}-\left(\begin{array}{l}
1 \\
0
\end{array}\right) V_{0}
$$

where $\omega_{0}$ and $V_{0}$ are angular velocity and velocity of the vehicle, respectively. To derive the dynamical property of information amount, time derivative of the $I_{L}$ is derived.

$$
\frac{d I_{L}}{d t}=\frac{S_{B} \dot{S}_{E}-\dot{S}_{B} S_{E}}{S_{E}^{2}}
$$

$S_{B}$ and $S_{E}$ are given in Eqs.(15) and (16). It is necessary to define the $x_{P}$ and $\theta_{P}$ of the focused area in order to derive the time derivative. The distance of focused area $x_{P}$ is proportional to the velocity of helicopter $V_{0}$.

$$
x_{P}=V_{0} \Delta t
$$


where $\Delta t$ is a constant with the unit of time. This shows that the vehicle moves the distance of $x_{P}$ in the period of $\Delta t$. The angle $\theta_{P}$ is set as constant, thus the vehicle focuses wide area in case of high speed.

The time derivatives of the $S_{B}$ and $S_{E}$ are derived.

$$
\begin{aligned}
\frac{d}{d t}\left(\begin{array}{l}
S_{B} \\
S_{E}
\end{array}\right) & =\left(\begin{array}{ll}
A & B \\
D & 0
\end{array}\right)\left(\begin{array}{c}
\dot{V}_{0} \\
V_{0} \omega_{0}
\end{array}\right)+\left(\begin{array}{l}
C \\
0
\end{array}\right) \\
& =\left(\begin{array}{ll}
A & B \\
D & 0
\end{array}\right)\left(\begin{array}{l}
a_{x} \\
a_{y}
\end{array}\right)+\left(\begin{array}{l}
C \\
0
\end{array}\right)
\end{aligned}
$$

where,

$$
\begin{aligned}
A & =V_{0}(\Delta t)^{2}\left(\tan \theta_{P}-\tan \theta_{A}\right) \\
B & =\left[-x_{A} y_{A}\left(\tan \theta_{P}-\tan \theta_{A}\right)+\frac{1}{2}\left(x_{P}^{2}-x_{A}^{2}\right)\left(1+\tan ^{2} \theta_{A}\right)\right] / V_{0} \\
C & =\left[x_{A}^{2}\left(\tan \theta_{P}-\tan \theta_{A}\right)-\frac{1}{2}\left(x_{P}^{2}-x_{A}^{2}\right) \tan \theta_{A}\right] V_{0} / x_{A} \\
D & =2 V_{0}(\Delta t)^{2} \tan \theta_{P}
\end{aligned}
$$

$a_{x}$ and $a_{y}$ are horizontal acceleration in the body fixed frame. Substituting Eq. (21) into Eq.(19), the time derivative of $I_{L}$ is given.

$$
\frac{d I_{L}}{d t}=E \cdot a_{x}+F \cdot a_{y}+G
$$

where,

$$
\begin{aligned}
& E=\left(S_{B} D-S_{E} A\right) / S_{E}^{2} \\
& F=-B / S_{E} \\
& G=-C / S_{E}
\end{aligned}
$$

Therefore, the information amount of safety can be changed by $a_{x}$ and $a_{y}$. This shows that the direction of acceleration changes the information amount. The information gathering control law is required to keep the information amount higher than the specified value $I_{R}$. Thus the shortage of information amount is defined in the following equation.

$$
I_{E}=\left\{\begin{array}{cc}
I_{R}-I_{L} & \left(I_{R}>I_{L}\right) \\
0 & \left(I_{R} \leq I_{L}\right)
\end{array}\right.
$$

In the case of $I_{L}$ is less than $I_{R}$, the controller is required to increase $I_{L}$. On the other hand, in the case of $I_{L}$ is greater than $I_{R}$, the high decreasing rate of $I_{L}$ is not desired. Therefore the following control law satisfies the both cases.

$$
\dot{I}_{L} \geq\left(\dot{I}_{L}\right)_{\min }
$$

where,

$$
\left(\dot{I}_{L}\right)_{\min }=k_{E}\left(I_{R}-I_{L}\right)
$$


$k_{E}$ is a feedback gain. The left hand side of Eq. (26) is given in Eq. (23). Equation (23) has two input variables, $a_{x}$ and $a_{y}$. Thus the minimum norm of input vector is chosen.

$$
\left(a_{x}\right)_{I}=E \cdot u_{N},\left(a_{y}\right)_{I}=F \cdot u_{N}
$$

where,

$$
u_{N}=\left(k_{E}\left(I_{R}-I_{L}\right)-G\right) /\left(E^{2}+F^{2}\right)
$$

This control law uses the same feedback gain in the cases of $I_{R}>I_{L}$ and $I_{R}<I_{L}$. The vehicle is required to keep the desired velocity and direction when the information amount satisfies the requirement. In this case, the following feedback law is used.

$$
\left(a_{x}\right)_{D}=k_{V}\left(V_{R}-V_{0}\right), \quad\left(a_{y}\right)_{D}=k_{\psi}\left(\psi_{R}-\psi_{0}\right) V_{0}
$$

where $k_{V}$ and $k_{\psi}$ are feedback gains. Finally, the information gathering control law is given as follows.

i.

$$
\begin{gathered}
E \cdot\left(a_{x}\right)_{D}+F \cdot\left(a_{y}\right)_{D}+G \geq\left(\dot{I}_{L}\right)_{\min } \\
a_{x}=\left(a_{x}\right)_{D}, \quad a_{y}=\left(a_{y}\right)_{D}
\end{gathered}
$$

ii.

$$
\begin{gathered}
E \cdot\left(a_{x}\right)_{D}+F \cdot\left(a_{y}\right)_{D}+G<\left(\dot{I}_{L}\right)_{\min } \\
a_{x}=\left(a_{x}\right)_{I}, \quad a_{y}=\left(a_{y}\right)_{I}
\end{gathered}
$$

The $\dot{I}_{L}$ in the inequality conditions are given in Eq. (26). The control law is called Information Amount FeedBack (IAFB) for it feeds back the information amount as one of the parameters for information gathering.

\subsection{Simulation result of absolute position}

The simulation result using the collision avoidance control with IAFB is introduced. Two different cases of similation will be shown in this scetion. The first case is the case with the helicopters. The velocity of the vehicle can be changed directly by the control law. The second case is the case with the fixed wing aircraft. The input is given as the angular velocity and the velocity itself is kept as constant.

\subsubsection{Simulation result of helicopters}

Figure 14 shows the initial condition of the evader and intruder. The intruder cannot be seen from evader at beginning of the control. The intruder is incoming from behind the obstacle with velocity of $10[\mathrm{~m} / \mathrm{s}]$ and $20[\mathrm{~m}]$ away from the obstacle. The evader starts from $150[\mathrm{~m}]$ away from the obstacle with various position defined by $y(0)$. Figure 15 shows the simulation result of the avoidance for different initial conditions. Figure 16 shows the case without IAFB for comparison. 


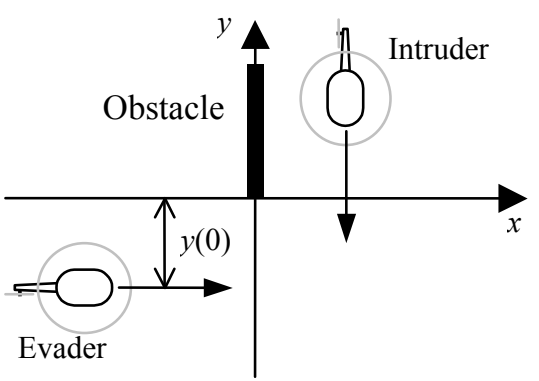

Fig. 14. Initial condition of evader and intruder.

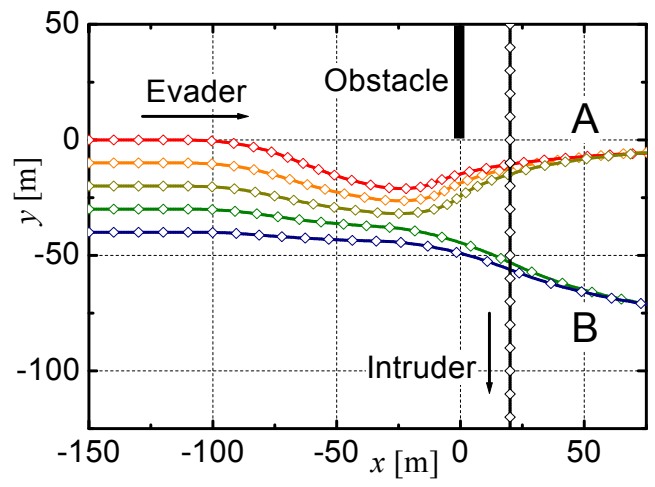

Fig. 15. Trajectory of evader with IAFB starting from different initial positions.

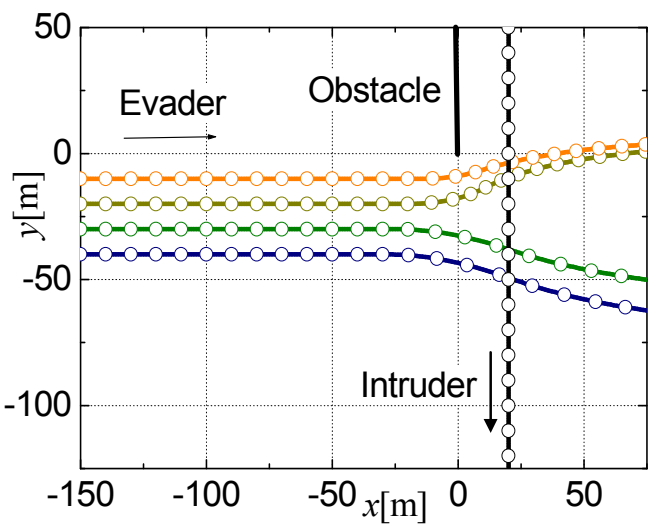

Fig. 16. Trajectory of evader without IAFC starting from different initial positions. 
In the first half of the control, the evader starts to obtain the information behind the obstacle. After the intruder is found approaching, the evader decides to evade either in front or back of the intruder depending on the estimated trajectory of the intruder passing in the way. In this case, the results were split into exactly two groups where evader decelerates and passes behind the intruder or accelerates and passes in front of intruder. In Fig.16, the case without IAFB, the first half of the information gathering does not occur, so the helicopter avoids the intruder after they find the incoming vehicle. Figure 17 is comparison of the minimum distance when the two vehicles pass each other. The result with IAFB shows higher level of avoidance due to the earlier motion of gathering information which leads to easier avoidance and faster recognition of the intruder.

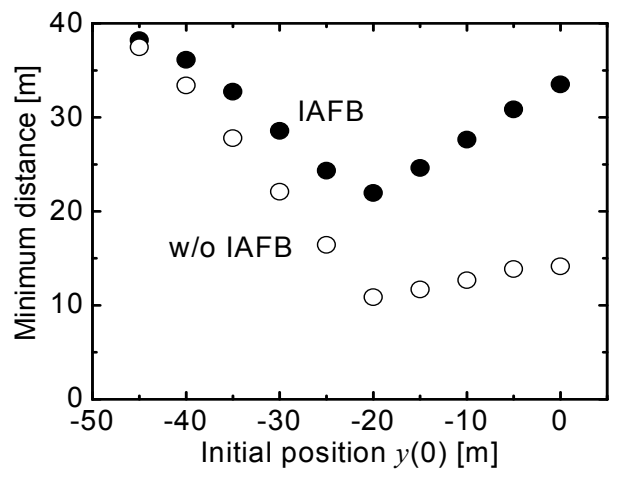

Fig. 17. Trajectory of evader without IAFC starting from different initial positions.

\subsubsection{Simulation result of fixed wing aircraft}

For fixed wing type aircraft, it is not easy and efficient to change the velocity so often. The control input for these types are changed to angular velocity input. Basic input is same as the one described in section 5.1. Most of the conditions are same as that of the case of the helicopters except that the cruising speed of evader and intruder is $100[\mathrm{~m} / \mathrm{s}]$ and the results are compared with different $I_{R}$ and different course of the intruder.

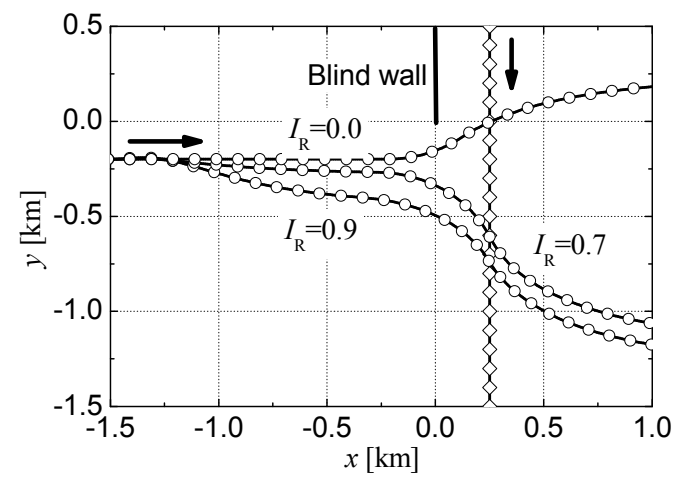

Fig. 18. Trajectory of evader with different $I_{R}$. 
Figure 18 shows the trajectory of evader with different $I_{R}$, in the case of $I_{R}=0$ this is same as the case without IAFB, we can see that the trajectory changes by the amount of information required.

Figure 19 shows the time history of the relative distance of the vehicles. The results show that the relative distance decreases very quickly in the case without IAFB and the minimum distance between the vehicles are shorter than the others. This clearly shows the effectiveness of the IAFB.

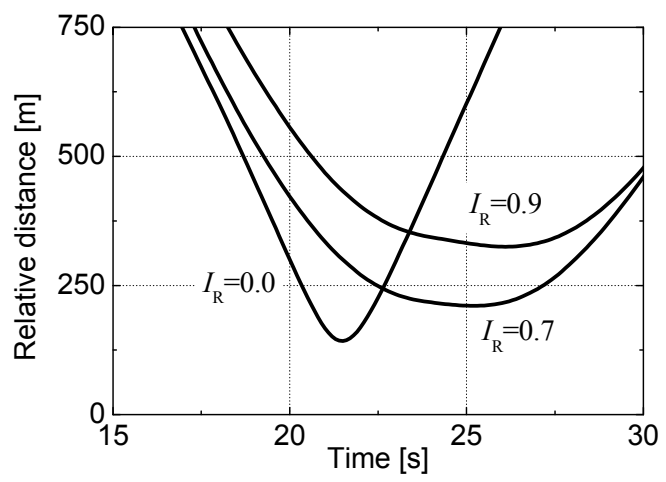

Fig. 19. Time history of relative distance between intruder and evader with different $I_{R}$.

Figure 20 shows the trajectory of evader when the intruder starts from different positions. The $I_{R}$ is set as 0.9 for this simulation. In the cases when the intruder is far from the obstacle, the trajectory is smoother because the intruder is found quicker. The other two cases makes sharp turns due to the slower finding of the intruder.

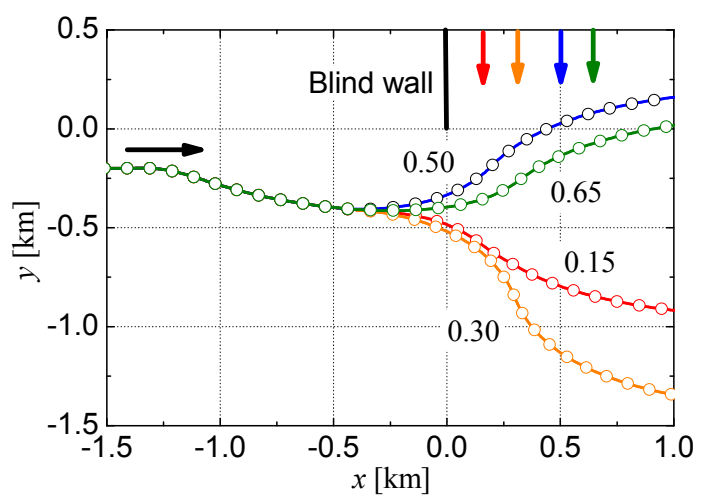

Fig. 20. Trajectory of evader when the intruder approaches from different course.

\section{Conclusion}

Two cases of collision avoidance control is simulated to see the effect of the information amount as parameter for control. One was that uncertainty of the information changes by the relative position of the evader and the target and the other was that uncertainty of the 
infomation is given as absolute position. Both cases have shown smoother and safer trajectories than the conventional control laws. The simulation results have shown that the control laws using information amounts does not rely on the coodinates. The motion of the aircraft show similar trajectories to that of humans to obtain safe margin to gain information when they do not have enough information.

\section{References}

Ciletti, M.D.; Meza, A.Z. \& Takushoku, S. (1997). Collision Avoidance Maneuver for Ships, Navigation - Japan Institute of Navigation-, Vol. 54, pp.83-89.

Frazzoli, E.; Mao, A.H.; Oh, J.H. \& Feron, E. (2001), Resolution of Conflicts Involving Many Aircraft via Semidefinite Programming, Journal of Guidance, Control, and Dynamics, Vol. 24, No. 1, pp.79-86.

Gates, D.J. (2009) Properties of a Real-Time Guidance method Preventing a Collision, Journal of Guidance Control and Dynamics, Vol. 32, No. 3, pp. 705-716.

Hiraoka, T.; Tanaka, M.; Kumamoto, H.; Izumi, T. \& Hatanaka, K. (2009a), Collision Risk Evaluation Index Based on Deceleration for Collision Avoidance (First Report) : Proposal of a new index to evaluate collision risk against forward obstacles, Review of Automotive Engineering, Vol. 30, No. 4, pp. 429-437.

Hiraoka, T.; Tanaka, M.; Takeuchi, S.; Kumamoto, H.; Izumi, T. \& Hatanaka, K. (2009b), Collision Risk Evaluation Index Based on Deceleration for Collision Avoidance (Second Report) : Forward obstacle warning system based on deceleration for collision avoidance, Review of Automotive Engineering, Vol. 30, No. 4, pp.429-447.

Iwama K. (2008), Study on Collision Avoidance Control Law using Information Amount Feedback, Master Thesis of Yokohama National University.

Kubota, N. \& Fukuda, T. (1999). An Intelligent Robotic System Based on a Fuzzy Approach, Proceedings of the IEEE, Vol. 87, No. 9, pp.1448-1470.

Miele, A.; Wang, T.; Mathwig, J.A. \& Ciarcia, M. (2010), Collision Avoidance for an Aircraft in Abort Landing : Trajectory Optimization and Guidance, Journal of Optimization Theory and Applications, Vol. 146, No. 2, pp.233-254.

Shioiri, H. \& Ueno, S. (2004), Three-dimensional Collision Avoidance Control Law for Aircraft using Risk Function and Fuzzy Logic, Transactions of JSASS, Vol. 46, No. 154, pp. 253-261.

Shioiri, H. \& Ueno, S. (2004), Collision Avoidance Control Law for Aircraft under Uncertain Information, Transactions of JSASS, Vol. 47, No. 157, pp. 209-215.

Slater, G.L.; Byram, S.M. \& Williams, T.W. (2006), Collision Avoidance for Satellites in Formation Flight, Journal of Guidance, Control, and Dynamics, Vol. 29, No. 5, pp. 11401146.

Stipanovic, D.M.; Hokayem, P.F.; Spong, M.W. \& Salijak D.D. (2007), Cooperative Avoidance Control for Multiagent Systems, Journal of Dynamic Systems Measurement and Control-Transactions of the ASME, Vol. 129, No. 5, pp.699-707.

Introduction to TCAS II (1990), U.S. Department of Transportation, Federal Aviation Administration. 


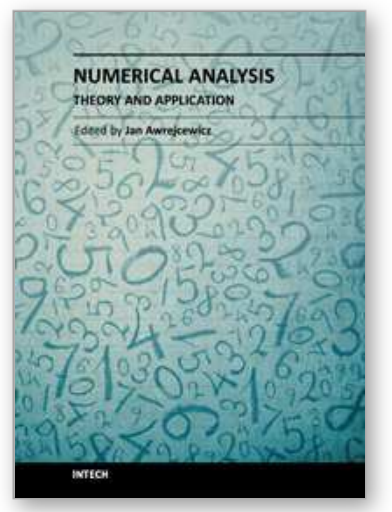

\author{
Numerical Analysis - Theory and Application \\ Edited by Prof. Jan Awrejcewicz
}

ISBN 978-953-307-389-7

Hard cover, 626 pages

Publisher InTech

Published online 09, September, 2011

Published in print edition September, 2011

Numerical Analysis â€“ Theory and Application is an edited book divided into two parts: Part I devoted to Theory, and Part II dealing with Application. The presented book is focused on introducing theoretical approaches of numerical analysis as well as applications of various numerical methods to either study or solving numerous theoretical and engineering problems. Since a large number of pure theoretical research is proposed as well as a large amount of applications oriented numerical simulation results are given, the book can be useful for both theoretical and applied research aimed on numerical simulations. In addition, in many cases the presented approaches can be applied directly either by theoreticians or engineers.

\title{
How to reference
}

In order to correctly reference this scholarly work, feel free to copy and paste the following:

Seiya Ueno and Takehiro Higuchi (2011). Collision Avoidance Law Using Information Amount, Numerical Analysis - Theory and Application, Prof. Jan Awrejcewicz (Ed.), ISBN: 978-953-307-389-7, InTech, Available from: http://www.intechopen.com/books/numerical-analysis-theory-and-application/collision-avoidance-lawusing-information-amount

\section{INTECH}

open science | open minds

\section{InTech Europe}

University Campus STeP Ri Slavka Krautzeka 83/A 51000 Rijeka, Croatia Phone: +385 (51) 770447

Fax: +385 (51) 686166 www.intechopen.com

\section{InTech China}

Unit 405, Office Block, Hotel Equatorial Shanghai No.65, Yan An Road (West), Shanghai, 200040, China 中国上海市延安西路65号上海国际贵都大饭店办公楼405单元 Phone: +86-21-62489820

Fax: $+86-21-62489821$ 
(C) 2011 The Author(s). Licensee IntechOpen. This chapter is distributed under the terms of the Creative Commons Attribution-NonCommercialShareAlike-3.0 License, which permits use, distribution and reproduction for non-commercial purposes, provided the original is properly cited and derivative works building on this content are distributed under the same license. 